\title{
Early sepsis diagnosis by microfluid chip detection of CD64 biomarkers in patient blood samples
}

\author{
Jose Olascoaga MS, Ye Zhang MS, Amanda Venable MSN, John Griswold MD, Dimitri Pappas PhD
}

Sepsis is a systemic inflammatory response to invading microorganisms. This often leads to organ failure and death if the diagnosis and treatment are delayed. ${ }^{1}$ This diagnosis is responsible for 250,000 deaths in the U.S. annually, affecting 10 of every 1,000 hospitalized patients, with a mean mortality rate of $35 \% .{ }^{1}$ However, sepsis can be cured if presumptive treatment is started immediately, making early detection essential. Successful algorithms include broad spectrum antibiotics and aggressive volume resuscitation for organ survival. ${ }^{2}$ The key is a definitive diagnosis of sepsis, yet current methods require culture of the infectious agent, a process that takes at least two days and up to multiple weeks, in an aggressive condition that requires prompt treatment. ${ }^{2}$ Unfortunately, many of the clinical signs of sepsis prove nonspecific to the condition and could be due to a number of inflammatory responses in which no infectious agent is present. The administration of broad spectrum antibiotics in these situations could cause more harm to patients and increase the prevalence of microbial drug resistance. ${ }^{2}$ Ultimately, knowing the correct diagnosis is vital within the first several hours of symptom onset to avoid under and over diagnosis with subsequent untoward outcomes.

Scoring systems are commonly used in an initial attempt to diagnose sepsis and include SOFA, APACHE, MEDS, and qSOFA. ${ }^{3}$ These factor in different physiological parameters that track organ function and assess the likelihood of a patient's being septic. When these determine the presence of sepsis in a patient, the hospital will normally activate a Code Sepsis protocol, including antibiotics, fluids,

Corresponding author: Jose Olascoaga Contact Information: Jose.e.olascoaga@ttuhsc.edu DOI: $10.12746 /$ swrccc.v6i26.508 blood analyses, and medical personnel. But the sensitivity of these scoring systems vary according to the patient's own personal health history and can be confounded by organ disease that was present before the inflammatory episode. ${ }^{3}$ Therefore, the lack of sensitivity of these scoring systems can lead to overtreatment of patients for several hours, resulting in unnecessary side effects and the use of hospital resources. Specificity is also an issue with these scoring systems, and they can miss patients with sepsis. Multiple studies have questioned the validity of these scoring systems in definitively diagnosing sepsis. ${ }^{3}$ Another point of focus is diagnosing sepsis through the presence of plasma biomarkers, which are elevated in sepsis and potentially correlate with the level of sepsis and the ultimate prognosis. These include C-Reactive Protein, Procalcitonin, s-TREM-1, HMGB-1, and CD64, among many others. ${ }^{2,4}$ Several of these have displayed positive correlations, yet no pragmatic, routine protocol has been established for their use in the ICU.

Dr. Dimitri Pappas (Texas Tech University) and his lab applied the knowledge of these biomarker elevations in sepsis to the creation of a point-of-care device that could assist in the definitive diagnosis of this condition. The microfluidic devices, or so-called "Lab on a Chip," capture cells based on increased expression of CD25, CD64, and CD69 antigens. The chip uses a small (0.01-0.05 $\mathrm{mL}$ ) blood sample and can diagnosis sepsis with $98 \%$ accuracy in our clinical study. The biomarkers of interest relate to the upregulation of expression of certain white blood cell surface receptors. CD64 of neutrophils is a strong marker for sepsis diagnosis; CD25 and CD69 are upregulated in lymphocytes during infection and sepsis. While each individual parameter provides strong correlation to the degree of infection and sepsis, the combination of these different parameters, measuring the level of activity of cells from two different lineages of white blood cells could overcome the variations within each 
cell line and provide better sensitivity and specificity than either one alone.

With the widespread use of microfluidic technologies for liquid biopsies and other bedside testing, Dr. Pappas and colleagues applied the predictive ability of CD marker expression to a precise and accessible device that could calculate these values at the point of care. These devices are made in a manner similar to semiconductor chip lithography. The microfluid chip design was initially printed at high resolution and a mold was created out of this design into a wafer using photolithography. A transparent elastic polymer is cast onto the mold to create channels for fluid control and cell capture. The chip is then assembled and coated with antibodies for CD25, CD64, and CD69. Blood samples are processed through the chip, and cells that are positive for each antigen are captured in different sections of the chip. Light microscopy readout of the chip yields cell counts for each antigen type, which are then used to diagnose sepsis, follow treatment, and predict improvement in patient health. ${ }^{5}$

Before introducing blood into the chip, both patient and control group blood samples were lysed by mixing with deionized water and then centrifuged, after which the leukocyte layer of each sample was separated and resuspended to its original concentration. These lysed leukocyte samples were pumped into the chip, allowed to settle for thirty minutes, and the remaining unbound cells were rinsed out with 3\% BSA in PBS solution. The remaining cells were analyzed and enumerated, providing a numerical value for each CD marker via antibody binding, and a threshold value was established for sepsis.

After demonstrating successful use of the chip in the laboratory, the next step was to check its effectiveness in the clinical setting. With a grant from The CH Foundation (Lubbock, Texas), Dr. Pappas, Dr. John Griswold (Texas Tech University Health Sciences Center) and their research team conducted a prospective clinical study at the University Medical Center (UMC) in Lubbock, Texas. Forty patients with suspected sepsis were enrolled into the study after giving informed consent and provided samples of plasma for analysis by the microfluidic chip to obtain
CD marker information. Ten volunteers also provided blood samples to serve as controls. Early trials of the chips displayed very positive results, but statistical analysis of the full dataset remains a work in progress. Because the chip provides a numerical value for $C D$ Marker expression, the research team can identify factors beyond just the diagnosis of sepsis. The data detected by the chip could provide predictive information on sepsis progression and prognosis, while taking into account patient demographics and other categories that the current UMC study is analyzing.

Our pilot study will not be the last in assessment of the device. Its correlation and accuracy will need to be validated by a larger data set, in different settings, and under different conditions. Full studies regarding the comparison of this device to established clinical methods are also necessary to evaluate how much of an improvement it provides in diagnosis. Meanwhile, Dr. Pappas has filed a patent for the chip, and he plans to make improvements to optimize its function. Ultimately, a prognostic device with this high level of accuracy, while remaining accessible, timely, and affordable, could be a powerful tool for hospitals and intensive care units, which frequently manage sepsis. The ability to quickly and accurately determine if a patient's condition is due to sepsis or not could potentially expedite treatment and save patient lives, while preventing the overuse of antibiotics and the rise of microbial drug resistance.

Keywords: Sepsis, microfluid assay, CD64, CD69, CD25.

From: Department of Chemistry (YZ, DP), Texas Tech University, Lubbock, TX; Department of Surgery (JG) and School of Medicine (JO), Texas Tech University Health Sciences Center, Lubbock, TX; University Medical Center (AV), Lubbock, TX

Submitted: $10 / 9 / 2018$

Accepted: 10/11/2018

Conflicts of interest: none

Funded: CH Foundation, Lubbock, Texas

This work is licensed under a Creative Commons Attribution-ShareAlike 4.0 International License 


\section{REFERENCES}

1. Polat G, Ugan R, Cadirci E, et al. Sepsis and septic shock: current treatment strategies and new approaches. The Eurasian $\mathrm{J}$ Medicine 2017;49:53-58.

2. Mat-Nor M, Ralib A, Abdulah N, et al. The diagnostic ability of procalcitonin and interleukin-6 to differentiate infectious from noninfectious systemic inflammatory response syndrome and to predict mortality. J Critical Care 2016;33: 245-251.

3. Seymour C, Liu V, Iwashyna T, et al. Assessment of clinical criteria for sepsis for the Third International Consensus
Definitions for Sepsis and Septic Shock (Sepsis-3). J American Medical Association 2016;315(8):762-774.

4. Gámez-Díaz L, Enriquez L, Matute J, et al. Diagnostic Accuracy of HMGB-1, s-TREM-1, and CD64 as markers of sepsis in patients recently admitted to the emergency Department. Academic Emergency Medicine 2011;18(8):807-815.

5. Zhang Y, Zhou Y, Li W, et al. Multiparameter affinity microchip for early sepsis diagnosis based on CD64 and CD69 expression and cell capture. Analytical Chemistry 2018;90: 7204-7211. 